\title{
BiOnto: An Ontology for Sustainable Bioeconomy and Bioproducts
}

\author{
Chiara Bicchielli, Noemi Biancone *, Fernando Ferri and Patrizia Grifoni \\ Consiglio Nazionale delle Ricerche, 00185 Rome, Italy; chiara.bicchielli@irpps.cnr.it (C.B.); \\ fernando.ferri@irpps.cnr.it (F.F.); patrizia.grifoni@irpps.cnr.it (P.G.) \\ * Correspondence: noemi.biancone@irpps.cnr.it
}

Citation: Bicchielli, C.; Biancone, N.; Ferri, F.; Grifoni, P. BiOnto: An Ontology for Sustainable Bioeconomy and Bioproducts. Sustainability 2021, 13, 4265. https://doi.org/10.3390/ su13084265

Academic Editor: Andreas Pyka

Received: 9 March 2021

Accepted: 8 April 2021

Published: 12 April 2021

Publisher's Note: MDPI stays neutral with regard to jurisdictional claims in published maps and institutional affiliations.

Copyright: (c) 2021 by the authors. Licensee MDPI, Basel, Switzerland. This article is an open access article distributed under the terms and conditions of the Creative Commons Attribution (CC BY) license (https:// creativecommons.org/licenses/by/ $4.0 /)$.

\begin{abstract}
Sustainable bioeconomy and circular economy are more and more connected to sustainable development goals. This requires engaging all the different stakeholders to promote peaceful and inclusive societies for sustainable development. Therefore, information access is a key challenge related to all the sustainable development goals. This article considers ideas, approaches and concepts related to sharing knowledge on Bioeconomy and collaborative ecosystems based on an ontology, aiming to facilitate information and services access. This ontology has been defined starting from the experience of the BIOVOICES project and from the need to establish a common terminology shared among scientists, enterprises, policymakers and civil society organisations on the bioeconomy. Indeed, the ontology provides a structured information of the BIOVOICES multi-stakeholders social platform's content, facilitating accessing and sharing it. The building process and the validation of the ontology have been described.
\end{abstract}

Keywords: bioeconomy; sustainable development goal; ontology

\section{Introduction}

The use of renewable resources represents a key challenge for sustainable development, i.e., for meeting "the needs of the present without compromising the ability of future generations to meet their own needs" [1]. "The term bioeconomy means using renewable biological resources from land and sea, like crops, forests, fish, animals and micro-organisms to produce food, materials and energy" [2]. In this perspective, the bioeconomy has all the characteristics for accelerating the evolution toward a circular and low carbon economy.

In 2015 the authors of the Global Bioeconomy Summit [3] already underlined that technological and social innovations are key elements; thus, it is necessary to involve all the actors. The crosscutting nature of bioeconomy offers a unique opportunity to address in a holistic perspective societal challenges such as food security, natural resource scarcity, fossil resource dependence and climate change, addressing at the same time sustainable environmental and economic development [4]. It implies the active collaboration of a broad range of stakeholders, including industry players, public authorities, researchers and civil society, i.e., the Quadruple Helix $(\mathrm{QH})$ stakeholders.

Linser and Lier [5] analysed the coverage of SDG-related issues with the different countries' bioeconomy strategies and observed a matching of up to 14 of the 17 SDGs.

The following SDGs 1-No poverty, 2-Zero hunger, 3-Good health and wellbeing, 4-Quality education, 8-Decent work and economic growth, 11-Sustainable cities and communities are related to the social and economic dimensions. The SDGs 6-Clean Water and Sanitation, 13-Climate Action, 14-Life below Water and 15-Life on Land are related to the environmental sustainability of the bioeconomy. The SDGs 7-Affordable and Clean Energy, 9-Industry, Innovation and Infrastructure, 12-Responsible Consumption and Production and 17-Partnerships for the goals are mainly related to the environmental and economic dimensions of the bioeconomic production of goods and energy. 
Although a larger market size of Bio Based Products (BBP) would contribute to a more sustainable society, it is crucial to accelerate the Transition towards a European bio-based economy and, in particular, toward a bio-based sustainable economy; in this perspective the involvement of all the $\mathrm{QH}$ actors should be strengthened.

A real development of the bioeconomy, as a driving force for sustainability, requires an effort to promote education and training, as well as public engagement and accessibility to information, products and services.

The BIOVOICES H2020 project (https: / / www.biovoices.eu/, accessed on 30 March 2021), funded by the European Commission, aims to ensure all the QH stakeholder groups' engagement involving a plurality of voices with different perspectives, knowledge and experiences. The project has facilitated co-creation and mutual learning actions between QH stakeholders by using participatory approaches to share knowledge and strengthen collective awareness.

Due to the importance of cooperating, sharing ideas and content and making such content accessible to researchers, entrepreneurs, policymakers and the general public, it is necessary to provide tools for collecting data and facilitating access to information and services.

Information access is a key challenge related to all the sustainable development goals and mainly with SDG 16 "Promote peaceful and inclusive societies for sustainable development, provide access to justice for all and build effective, accountable and inclusive institutions at all levels".

This article considers ideas, approaches and concepts related to sharing knowledge on Bioeconomy and collaborative ecosystems based on an ontology, aiming to facilitate information and services access. This ontology has been defined starting from the experience of the BIOVOICES project and from the need to establish a common vocabulary among scientists, enterprises, policymakers and civil society organisations on the topics and the challenges related to the bioeconomy.

Any kind of facilitation for sharing information and services is also crucial for making the Transition to a Circular Bioeconomy Sustainable and Inclusive. Building an ontology can represent an important progress in this perspective.

First of all, we introduce some definitions of "ontology" given in the literature. Gruber (Gruber, 1993) defined ontology as "a specification of a conceptualisation". Guarino et al., (2009) explain that there are two meanings for "ontology". The first one refers to "a philosophical discipline, namely the branch of philosophy dealing with the nature and structure of reality". The second meaning refers to "an ontology as a special kind of information object or computational artefact". In particular, the activities of the BIOVOICES project refer to the second meaning. Building an ontology requires to "analyse relevant entities and organise them into concepts and relations" (Guarino et al., 2009).

Using ontologies allows to define a structured and universal domain of interest, through the definition of relations and pertinent concepts related to the domain itself. Moreover, ontologies are frequently used in web search engine: they allow to generate a semantically coherent output and, also, to extend the search to all the concepts semantically related to the query. Prananingrum et al. [6] proposed, for example, a system that uses an ontology, representing National Occupational Map of ICT domain, in order to generate a structured output based on ontology concepts. Kumar and Ramu [7] designed a system to add and search job opportunities using an ontology in order to specify professional figures, education levels etc.

Indeed, in BIOVOICES, the ontology organises and classifies concepts of information collected and shared in the social platform (https://www.biovoices-platform.eu, accessed on 30 March 2021) and relationships between these concepts, facilitating any activity of searching documents, events, finding and extracting any information of interest (see Figure 1). 


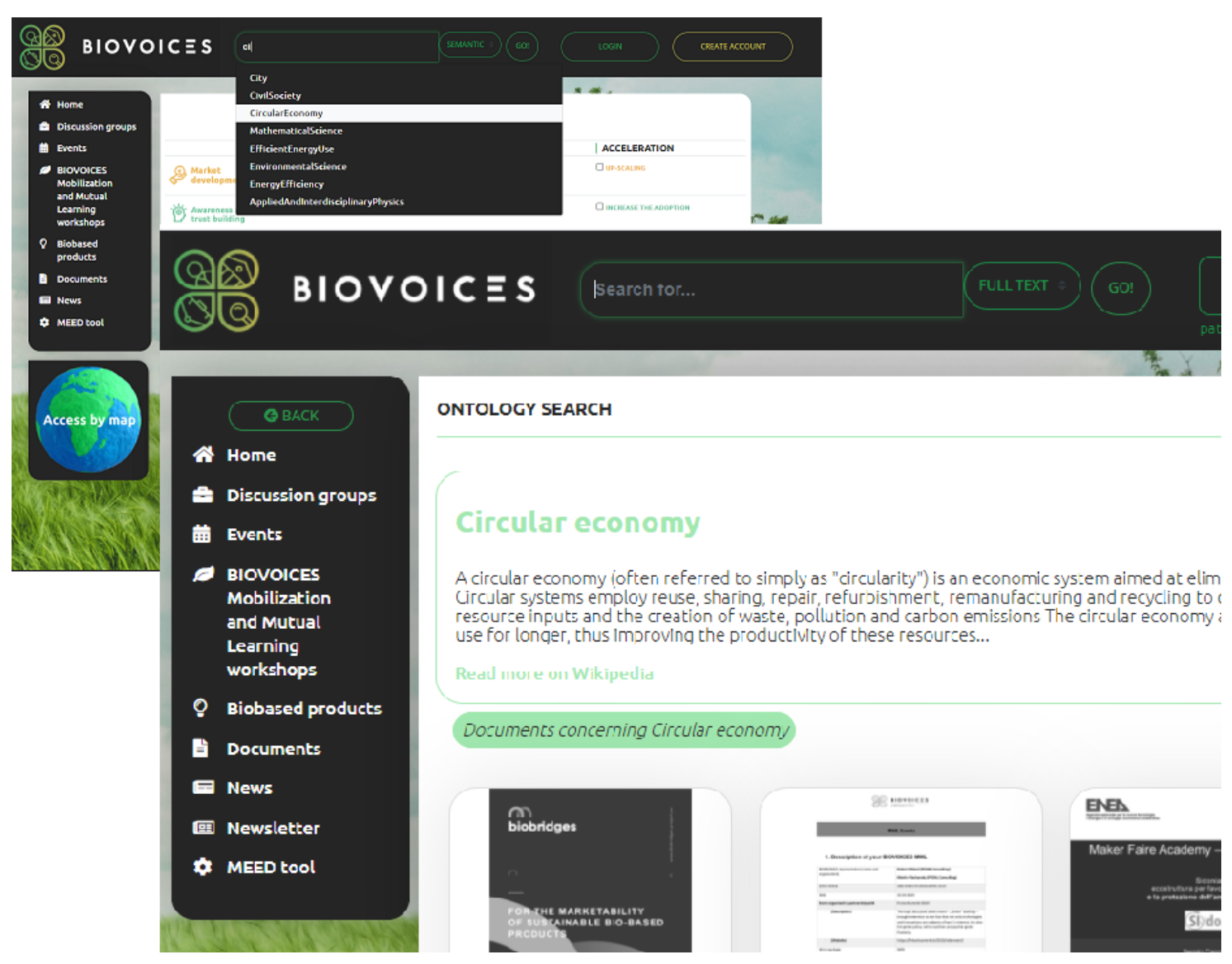

Figure 1. An example of searching by ontology concepts in the BIOVOICES platform.

Sharing content in the BIOVOICES social platform will enrich the discussion. This social platform can be used by all the stakeholders also not involved in the BIOVOICES project, for sharing their events, information and knowledge, discussing the emerging key issues related to the bioeconomy and promoting the transition toward the concept of sustainable bioeconomy.

Concepts contained in these contents and their relationships have been selected and organised in an ontology. Using ontologies entails many advantages: first of all, providing structured information, it is possible to obtain a formal and unambiguous specification of the domain of interest. This reduces the risks of misinterpretation and misunderstanding for users who are not familiar with the domain itself. Moreover, ontologies implemented through a standard language are interoperable, integrable, re-usable and portable. If ontologies are developed upon Semantic Web technology they are intrinsically enabled in the Semantic Web [8]. Finally, ontologies, provide an explicit knowledge through the included concepts, but they can also provide implicit knowledge obtained through inference rules and reasoning processes. Automated reasoning is a key feature within knowledge-based systems [9].

The paper is structured as follows: the second section introduces information related to the BIOVOICES project and a sketch of the main concepts of the Ontology for Bioeconomy; the third section describes the methodology used to define the taxonomy and to build the Ontology. Section 4 describes the validation of the ontology. Section 5 describes the reasoning process and the search system designed through the use of the developed ontology. Finally, Section 6 concludes the article. 


\section{Background}

\subsection{The Context}

As described in the previous sections, the BIOVOICES project (https: / / www.biovoices. $\mathrm{eu} /$, accessed on 30 March 2021) aims to promote bioeconomy and a sustainable world complying with the emerging environmental, societal and economic challenges in Europe.

The BIOVOICES project encourages the quality, relevance, know-how and social acceptability of bio-based products. In particular, the project has promoted a broad discussion among the $\mathrm{QH}$ stakeholders to stimulate their active collaboration for making bioeconomy sustainable. Indeed, the bioeconomy needs the active cooperation of a broad range of stakeholders: industry, agriculture and business players, public authorities and policy makers, researchers and civil society. Those can be referred with the "quadruple helix model".

Many projects related to bioeconomy have been funded in the last five years at the European level. Some of them focus on engaging the different stakeholders in a large debate, understanding the sustainability features of bioeconomy and its connection with the circular, green and blue economy. We mention some of them only by way of example and without claiming completeness. Supporting the active engagement and interaction among all stakeholders was one of the main objectives in the project BIOBRIDGES (https:/ / cordis.europa.eu/project/id/792236, accessed on 30 March 2021), "aiming at boosting the marketability of bio-based products-BBPs by establishing close cooperation and partnership between bio-based Industries-BBI, brand owners and consumers' representatives".

The project STAR-ProBio (https:/ / cordis.europa.eu/project/id/727740, accessed on 30 March 2021) paid particular attention to the stakeholders' engagement, considering the different perspectives of the bioeconomy sustainability, having between its purposes the development of a sustainability scheme.

The LIFT project (Unleash the potential of CSAs results to contribute to sustainable and competitive Bio-Based Industries in Europe) (https://cordis.europa.eu/project/id/837858, accessed on 30 March 2021) aimed to valorise the Coordination and Support Actions (CSAs) outcomes stimulating the collaboration among them.

The BIOVOICES project provided a social web-platform that allows managing and sharing discussions, Events, Mobilisation and Mutual Learning (MML) workshops and collections of Documents and sharing information related to bio-based products (https: / / www.biovoices-platform.eu, accessed on 30 March 2021), aiming to facilitate the engagement of the different stakeholders and their collaboration. In this perspective the platform provides a cross-cutting tool, also considering its participatory characteristics. Indeed, this platform facilitates the access to information, supporting knowledge sharing and co-building. The platform will be maintained active at least five years after the end of the project for enabling the stakeholders in debating, co-building and sharing contents.

The BIOVOICES social web platform allows users to play both the role of provider and consumer of resources (services, information and knowledge contributing to content enrichment or gleaning knowledge as a consumer). These stakeholders come from many different realities and they are part of a plurality of voices with different perspectives, knowledge and experiences. This requires establishing a common language and standardising the meanings of the terminology used. Thus, for facilitating access to the knowledge within the BIOVOICES social platform, the BIOVOICES ontology has been defined, formalised and implemented.

\subsection{A Sketch of the Main Concepts of the Ontology for Bioeconomy}

Our purpose is to provide a structured representation of knowledge related to the bioeconomy domain. The bioeconomy concept became relevant due to the promotion made by the European Commission to improve sustainability. Bioeconomy aims to a sustainable economic growth through the replacement of fossil-based resources by bio-based resources and the use of new biotechnologies. In the literature are not publicly available ontologies 
describing the bioeconomy domain, although some glossaries and key terms lists are provided $[10,11]$. The proposed ontology implements our reference model for bioeconomy concepts, which explicitly aims at sustainability, circular economy and environmental governance. "Bioeconomy is Europe's response to key environmental challenges the world is facing already today. It is meant to reduce the dependence on natural resources, transform manufacturing, promote sustainable production of renewable resources from land, fisheries and aquaculture and their conversion into food, feed, fibre, bio-based products and bio-energy, while growing new jobs and industries." [12]. Following the European Commission definition, we designed the ontology entity for "Bioeconomy" including the concept of "Sustainability": these concepts are closely linked in our model. "Sustainability" could be reached through a sustainable development that aims to meet the needs of present generations without compromising future generations' ability to meet their own needs. In other words, establishing an "Environmental governance", which includes local and global actions, regards social, economic and environmental issues and improves ecological policies. To make bioeconomy sustainable, a key aspect is applying circularity. "Circular economy" is an economic system which aims to retain the value of all resources in the economic cycle for as long as possible before these resources reach the end-of-life stage and supports a "Sustainable Bioeconomy". Since 1974, Hammack et al. [13] analysed the impact of management of the natural resources and proposed changes in the natural environment. Nowadays, the European Commission planned a Circular Economy strategy which consists in a set of measures limiting resources use and waste generation to help European businesses and consumers to make the transition to a more sustainable production and consumption. There are several business models developed to help in the transition to circular economy: for example, Núñez-Cacho et al. [14] provides a business model that helps in the transition to the Circular Economy, based on the theory of SocioEmotional Wealth (SEW). Górecki et al. [15] developed a concept framework to describe emerging circular economy business models and perform a simulation-based analysis to assess the possibility to adopt circular economy in construction companies.

Moreover, the ontology also includes the five macro areas (A-Market development, BAwareness and trust building, C-Supporting strategies, regulatory frameworks legislation and standards, D-Supporting environment (Infrastructures, intermediaries, new business opportunities), E-Regional/Local development) that group the 12 most relevant challenges addressed during the MML workshops according to the three development phases of innovation systems (1-Business case: Product is 95\% mature and becomes a business case, 2-Go-to-market: Product is mature and market increases to $5 \%$ among niche groups, 3-Acceleration: Market increases above and reaches new user groups), as defined in [16].

Figure 2 shows a subset that contains the previously described concepts with their relations. This is only a small part of the ontology's domain model we implemented. 


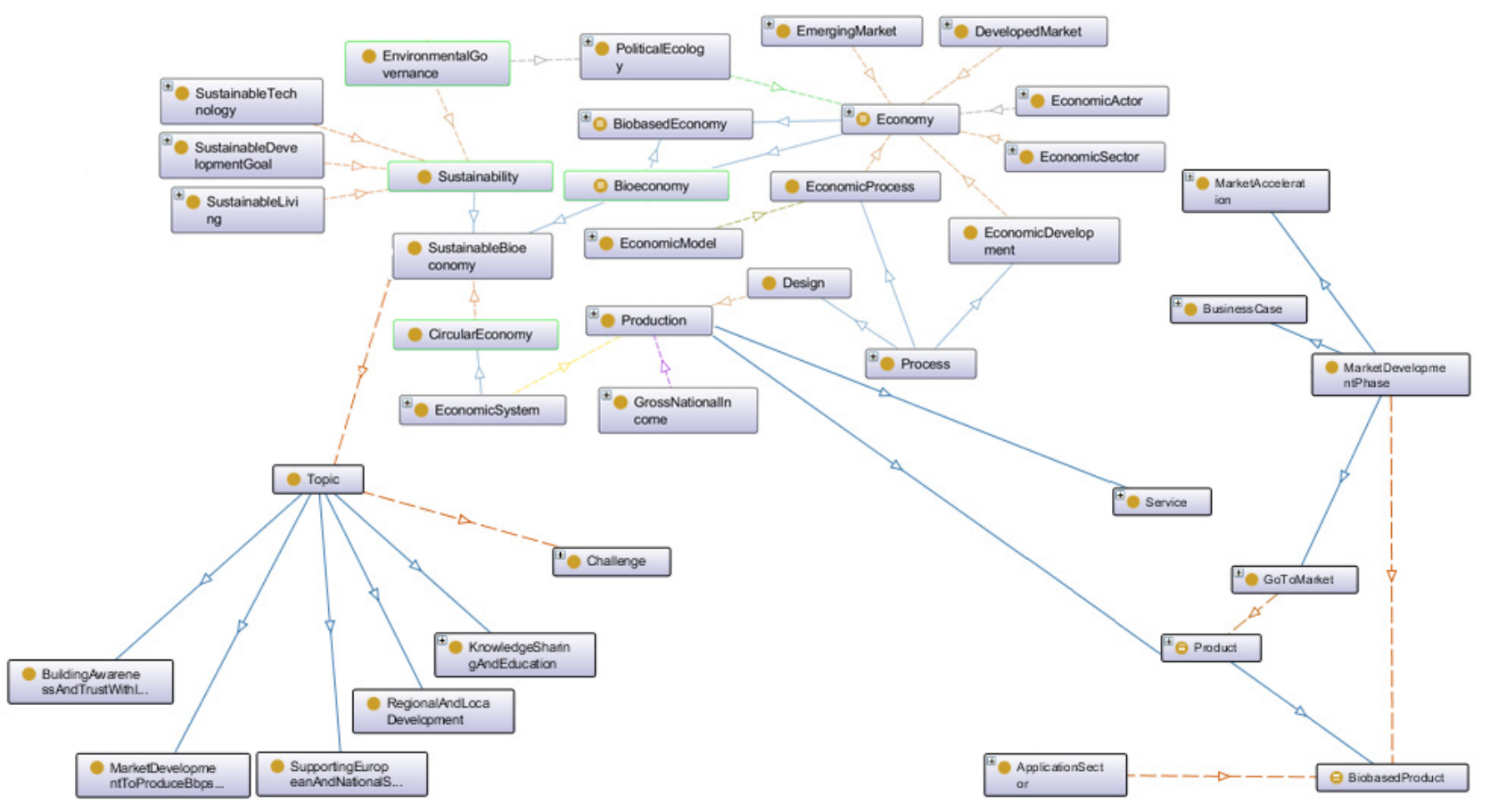

Figure 2. Subset of concepts from the implemented ontology.

\section{The Ontology Definition}

The BIOVOICES ontology building process consists of two steps:

- building a taxonomy;

- building the ontology, enriching the taxonomy.

A general procedure of an ontology construction has been adapted starting from the one proposed by Noy and McGuiness [17] and it consists of the following phases: (1) defining a set of criteria; (2) taxonomy construction; (3) ontology construction; (4) formal description; (5) defined classes creation; (6) reasoning process; (7) consistency verification; and (8) a set of results.

\subsection{BIOVOICES Taxonomy Construction}

This section describes the taxonomy building process, which consists of the three steps(A, B and C) synthesised in Figure 3.

The taxonomy construction was based on: (A) an in-depth analysis of selected documents on the bioeconomy themes. Moreover, we analysed the services to be included in the BIOVOICES multi-stakeholders' social platform, (B) the extraction of the main concepts from these documents, $(\mathrm{C})$ the definition of a set of hierarchical relationships among the concepts identified, which enabled us to build the taxonomy related to a first set of Bioeconomy concepts that are the basis for sharing knowledge and terms among the different stakeholders (see a sketch in Figure 3iii and concerning BIOVOICES social platform structure (see a sketch in Figure 4). Indeed, the platform enables all the different actors to organise Events, Mobilisation and Mutual Learning workshops, to share knowledge about the Bioeconomy topics, collect and access documents organising them in Collections of documents, etc. All these issues are included in the BIOVOICES social platform's taxonomy to facilitate accessing content and services. 


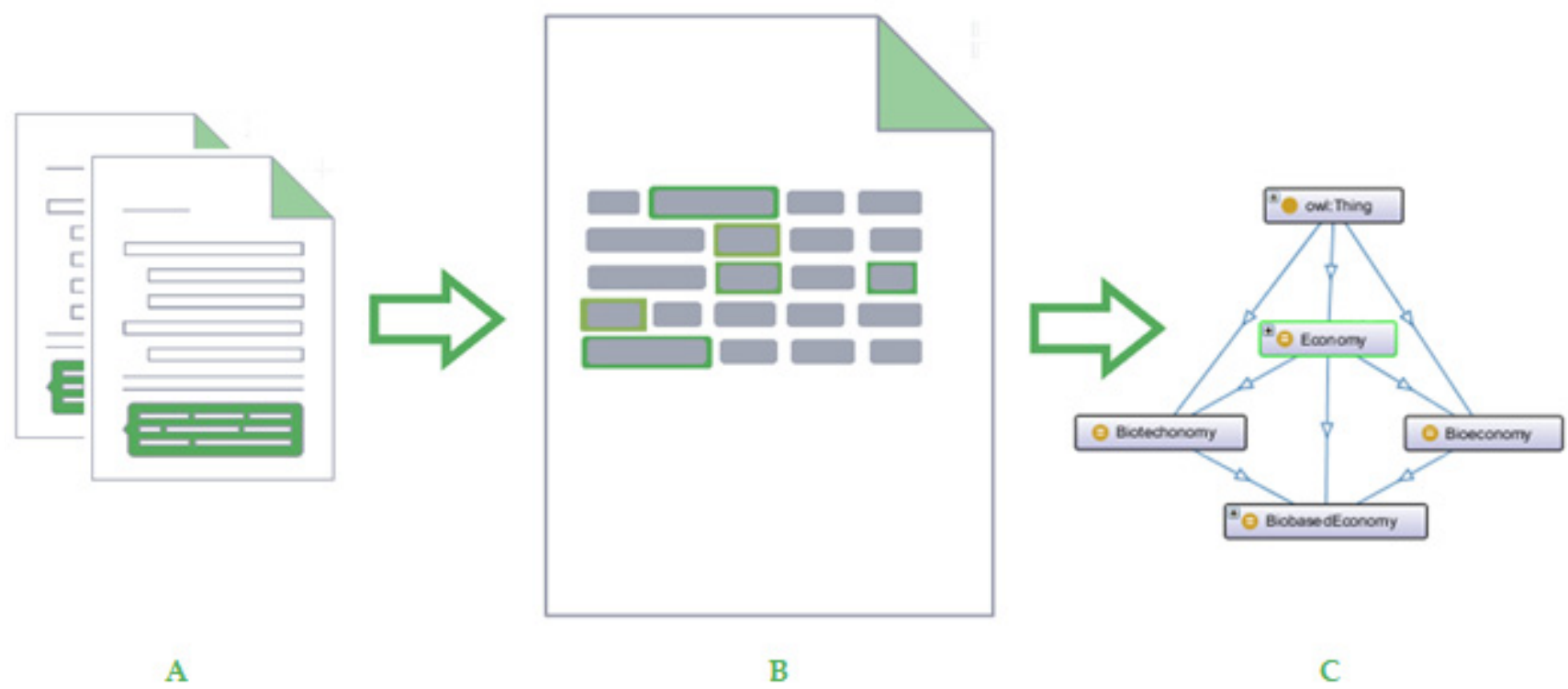

Figure 3. Taxonomy building process.

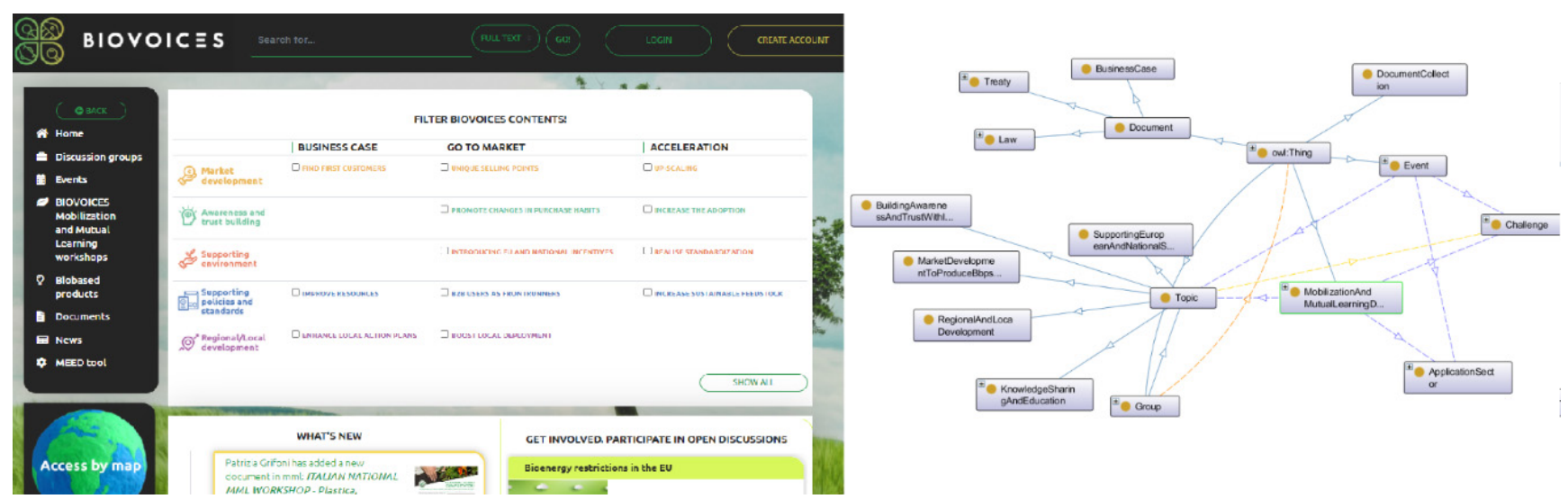

Figure 4. Subset of the BIOVOICES Taxonomy that structures the BIOVOICES multi-stakeholders' social platform.

The platform, indeed, is the primary tool of communication and collaboration for large communities. It contributes to establish common knowledge in an understandable way for all the QH actors; in this perspective, it is mandatory to establish common and transparent language. The BIOVOICES platform uses a participatory approach and each registered member can contribute to the online discussion and content. The administrator periodically checks the content. The set documents used to extract the first core of concepts for building the taxonomy consists of: (1) the deliverables produced by the BIOVOICES project in the first phase of the project, (2) the key documents on the bioeconomy produced by the European Commission (e.g., the EU Bioeconomy Strategy [18]) and 4 the open scientific documents selected (starting from 2017) using Google Scholar, Scopus and Web of Science (WoS) searching for "Bioeconomy", "Bioeconomy, strategy", "Bioeconomy, Circular economy" (see the menu "Documents" in Figure 4).

Other documents shared in the platform during the events, the Mobilisation and Mutual learning workshops or made available in the online libraries, have been included only in a second phase, i.e., in the ontology building step starting from the taxonomy.

The methodology adopted to construct the taxonomy makes use of different semiautomatic steps of selection and analysis of the content uploaded by users to the platform. The implementation of the different steps was supported by various frameworks, to obtain 
the collection of concepts to be added to the concepts related to the services of the platform, starting from a set of free text documents.

Text extraction was performed using the Aspose API: this suite allows the manipulation of different documents format and converts the document text in a stream of strings that could be used to perform the analysis of terms.

Text Razor API has been used to carry out a deep analysis of texts categorising terms relying on their semantic meaning. A set of concepts has been extracted from each document based on two scores: relevance and confidence.

Relevance is the pertinence that an entity has to the source text. This value is a float on a scale of 0 to 1 , with 1 that indicates the maximum value for relevance. Relevance is determined by the contextual similarity between the entity's context and facts in the TextRazor knowledgebase [19]. The confidence is the assurance that TextRazor has correctly evaluated an entity as valid. TextRazor uses an ever-increasing number of signals to help spot valid entities, all of which contribute to this score. These include the semantic agreement between the context in the source text and TextRazor knowledgebase, compatibility between other entities in the text, compatibility between the expected entity type and context, prior probabilities of having seen this entity across Wikipedia and other web datasets. The score is technically unbounded, but typically ranges from 0.5 to 10 , with 10 representing the highest confidence that this is a valid entity [19].

For example, analysing the document "Monitoring Bioeconomy Transitions with Economic-Environmental and Innovation Indicators: Addressing Data Gaps in the Short Term" [20], TextRazor is able to find the relevant entities and extract the context of the document itself.

Figure 5 represent a partial output of the API: for example, in this case, the "Sustainability" entity obtains the maximum value of relevance. In other words, we can assume that "Sustainability" is one of the main topics of this document. Furthermore, for the confidence score, TextRazor associates a high value to the concept of "Sustainable Development": this means that the previous entity is extremely pertinent to the analysed document.

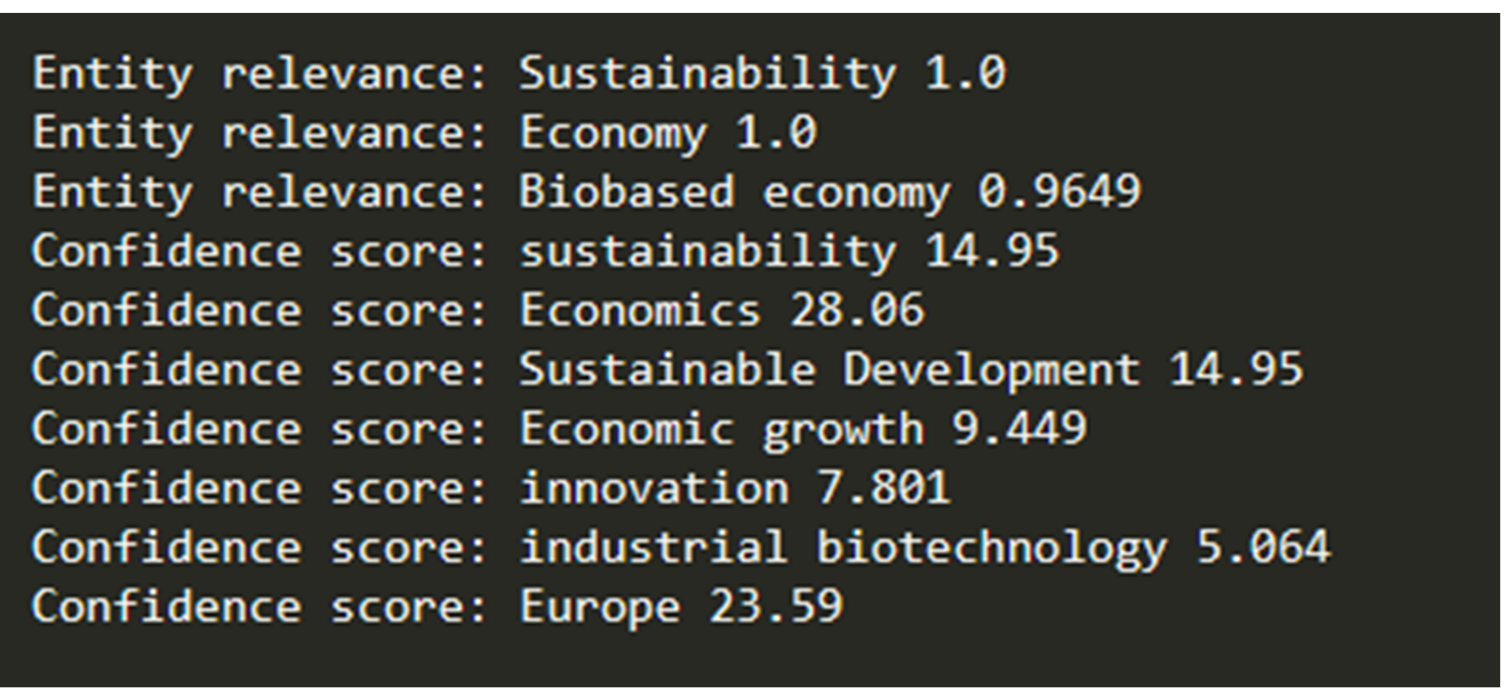

Figure 5. Partial output from TextRazor document analysis.

After some experiments, it has been chosen to assign a value of 0.5 to these two scores, because it was observed that with this tuning the methodology carried out a good classification.

After the concept identification hierarchical relationships among them are defined using Protegè [21] and formalised in OWL [22]. 


\subsection{Ontology Construction}

After building the BIOVOICES taxonomy, an in-depth analysis was carried out starting from information and knowledge produced and collected within some mutual and mobilisation learning workshops held within the BIOVOICES project activities and considering the documents and information uploaded on the BIOVOICES social platform. The analysis was carried out on a corpus consisting of 321 documents and information produced by 107 mobilisation and mutual learning events, 37 Discussion groups, 33 marketplace products.

The MML workshops were quadruple helix events organised at European, national and regional level. The organisers identified the topics mainly in connection with the challenges and the application sectors related to the bioeconomy and the biobased products, identified at the beginning of the BIOVOICES project [16] and shown in Table 1.

Table 1. Challenges and application sectors related to the bioeconomy and the biobased products.

\begin{tabular}{|c|c|}
\hline Challenges & Application Sector \\
\hline $\begin{array}{l}\text { A1-Find first customers } \\
\text { A2-Specify Unique Selling Points (USP) } \\
\text { A3-Up-scaling } \\
\text { B2-Changes in purchase habits } \\
\text { B3-Increase the Adoption } \\
\text { C2-Introduce EU and national incentives } \\
\text { C3-Release standardisation } \\
\text { D1-Improve the ecosystem to enhance } \\
\text { business cases } \\
\text { D2-B2B users as frontrunners } \\
\text { Other }\end{array}$ & $\begin{array}{l}\text { - } \quad \text { Cleaning and hygiene, personal care and } \\
\text { cosmetics, health and biomedical } \\
\text { - } \quad \text { Textile products, clothing, sports and toys } \\
\text { - } \quad \text { Food packaging, disposable tablewear } \\
\text { - } \quad \text { Biofuels and bioenergy } \\
\text { - } \quad \text { Building, construction and restoration, } \\
\text { paintings, decorations and furniture } \\
\text { Nutraceuticals, environmental } \\
\text { bioregulation and biological sensors } \\
\text { Other }\end{array}$ \\
\hline
\end{tabular}

The participation in the workshops was voluntarily based and the participants' registration was asked. The platform supports the organisation phase of MMLs and events and sharing any content and result.

As discussed earlier, building the ontology is an incremental process that begins with identifying the main concepts and relationships that define the taxonomy. The initial set of concepts was extended through an automated process and, the model has been furtherly enriched by extraction of all concepts and relationships to form the ontology's backbone.

The ontology has been built using FluentEditor, a tool based on a Controlled Natural Language approach [23]. We provide some examples to explain how we defined the semantic relationships between the concepts of the ontology.

Let us consider the class "Biobased Economy"; it takes a central place in the ontology (Figure 6). Through the use of grammar expression, in particular the rule "concept equivalency", implication states in concept subsumption. Through the following statements: "Something is a biobased-economy if-and-only-if-it is a bioeconomy" and "Something is a biobased-economy if-and-only-if-it is a biotechonomy" the ontology is defined specifying that the "Biobased Economy" class is equivalent to "Bioeconomy" and "Biotechonomy" classes. Moreover, the "Biobased Economy" concept has been designed as a subclass of the Economy concept through the rule: "Every biobased-economy is an economy". Indeed, the "concept subsumption" rule assumes that one concept subsumes the other one if the set described by the first concept is a subset of the other one. 


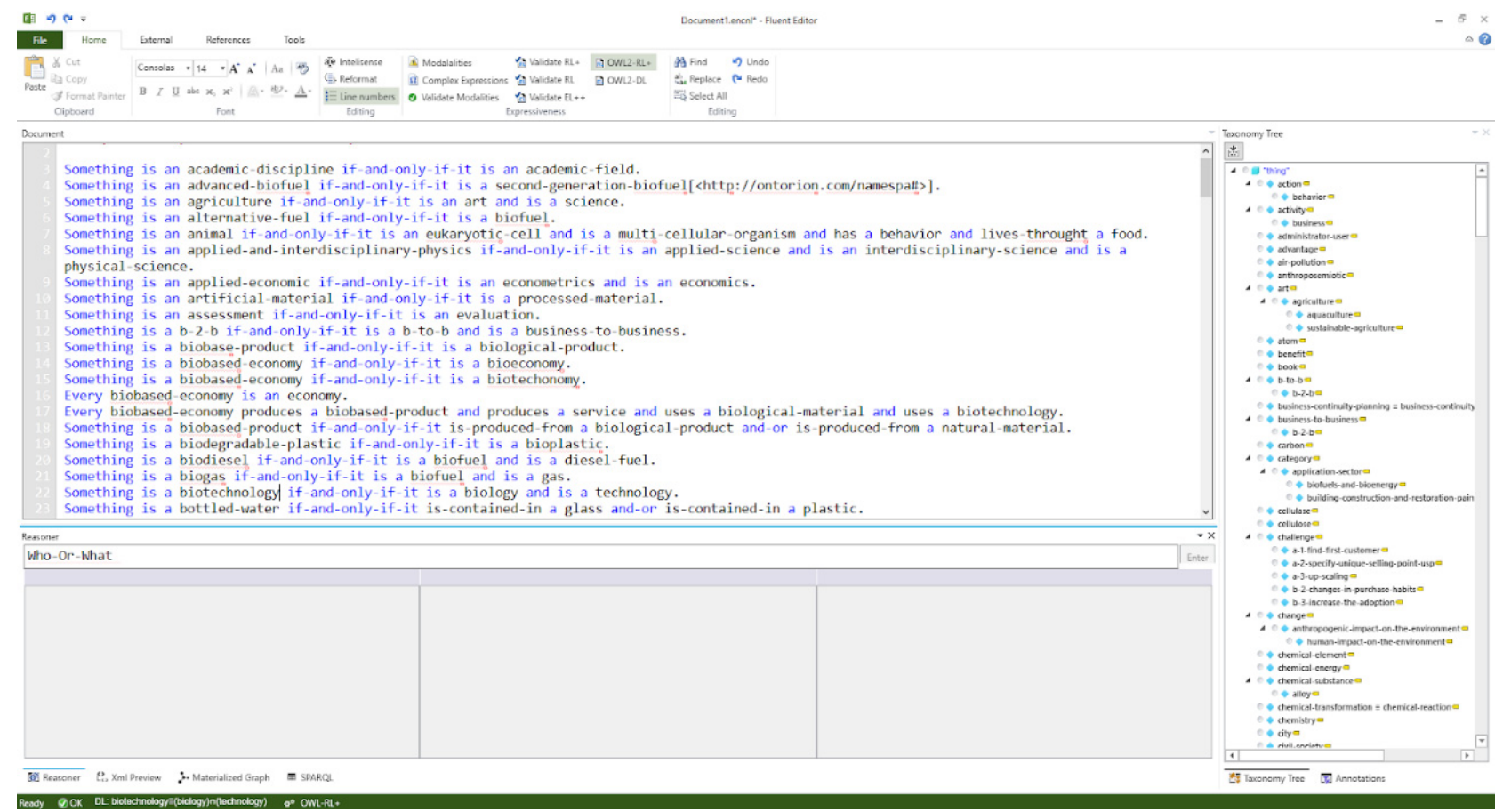

Figure 6. Ontology in FluentEditor.

To further enrich the "Biobased Economy" concept, some relations have been added. Every "biobased-economy" produces a "biobased-product" and produces a service and uses a "biotechnology". This means that the "Biobased Economy" concept has a direct connection with the concepts of "Biobased Product", "Biological Material" and "Biotechnology"; these connections are represented through the relations "produces" and "uses". Furthermore, the "Biobased Economy" concept has been enriched through the connection with the "Circular Economy" concept: these two entities are linked through the definition of "Sustainable Bioeconomy" that has been represented as the intersection between "Bioeconomy" and "Sustainability".

Once all the relationships between concepts have been defined through rules as the mentioned above, the ontology model has been formalised and an OWL (Ontology Web Language) file has been produced.

OWL [22] is a standard semantic web language widely used for ontology modelling and, through the use of this format, the resulting ontology, made by FluentEditor, can be loaded into Protégé (Stanford University, Stanford, CA, USA) [24], that also enables to have a deductive classification for the ontology and its visualisation. Figure 7 shows a graphical representation of a fraction of the ontology obtained by OntoGraph function in Protegè. 


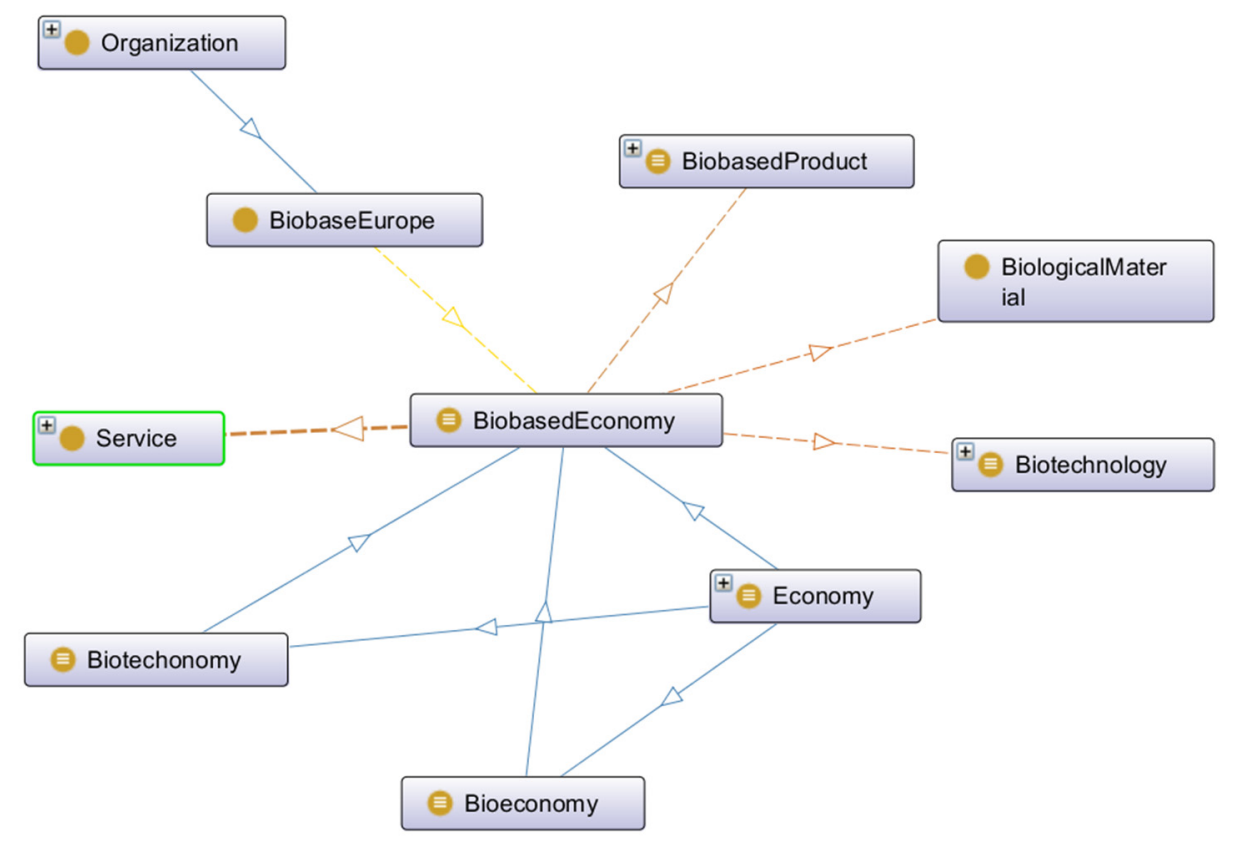

Figure 7. Ontology view by OntoGraph.

\section{Validation}

The ontology construction methodology provides as last steps the validation and evaluation. The main purpose is to check the formal structure of the proposed ontology and its coherence with the domain knowledge.

There are different approaches to validate and evaluate an ontology. Tartir et al. in [25] describe three different methodologies: evolution based, logical (rule-based) and metric-based (feature-based).

The evolution-based approach analyses the changes in an ontology over the time, using its different versions. These changes could be due to the evolution in the domain, in conceptualisation or the explicit specification. Through this approach, it is possible to find out errors or invalid changes.

The logical approach validates ontologies using rules included in the ontology language but also customises the rules defined by the designer. The metric-based approach collects a set of techniques that are used to provide a quantitative measure of the ontology quality. It also provides an analysis of the type of knowledge represented with the ontology through an inspection of classes' configuration within the ontology graph.

We used the logical approach to validate the BIOVOICES ontology combined with expert's validations. The evolution approach was not applicable to the use case because the BiOnto ontology was in its first version. The metric-based approach needs a comparison with other ontologies. In the literature, we did not find formalised and validated ontologies comparable with the proposed ontology. Therefore, a set of queries has been modelled and implemented using a Description Logic (DL) query mechanism to examine the proposed ontology's correctness.

Query's implementation and execution have been done through Protegè editor. It provides a module for DL queries and several reasoners to infer logical consequences from a set of asserted facts or axioms and to check their correctness.

Ontology validation is a process to assert the correctness and consistency of an ontology. This paragraph contains some examples of queries used to verify the accuracy in retrieving information with response to the user's needs and to check the correctness of the results.

In Figure 8, the following query has been executed:

Fuel and isProducedFrom some VegetableOil 


\section{Query (class expression)}

Fuel and isProducedFrom some VegetableOil

\section{Execute Add to ontology}

\section{Query results}

Equivalent classes $(0$ of 0$)$

Superclasses (6 of 6 )

Ð FossilGas

Fuel

Gas

@ Material

NaturalGas

owl:Thing

Direct superclasses (1 of 1 )

Fuel

Direct subclasses (1 of 1 )

FirstGenerationBiofuel

Subclasses (2 of 2)

FirstGenerationBiofuel

owl:Nothing

\section{Query for}

$\checkmark$ Direct superclasses

$\checkmark$ Superclasses

$\checkmark$ Equivalent classes

Direct subclasses

Subclasses

Instances

\section{Result filters}

Name contains

Display owl:Thing

(in superclass results)

Display owl:Nothing

(in subclass results)

Figure 8. First query output.

This query aims to find the relevant Fuel that fulfilled the feature of being produced using vegetable oil. The query output is compliant with the expected result: first-generation biofuels are made from sugar, starch, vegetable oil or animal fats using conventional technology [26].

In Figure 9 the following query has been executed:

Material and produces some Energy and reactsWith some ChemicalSubstance 


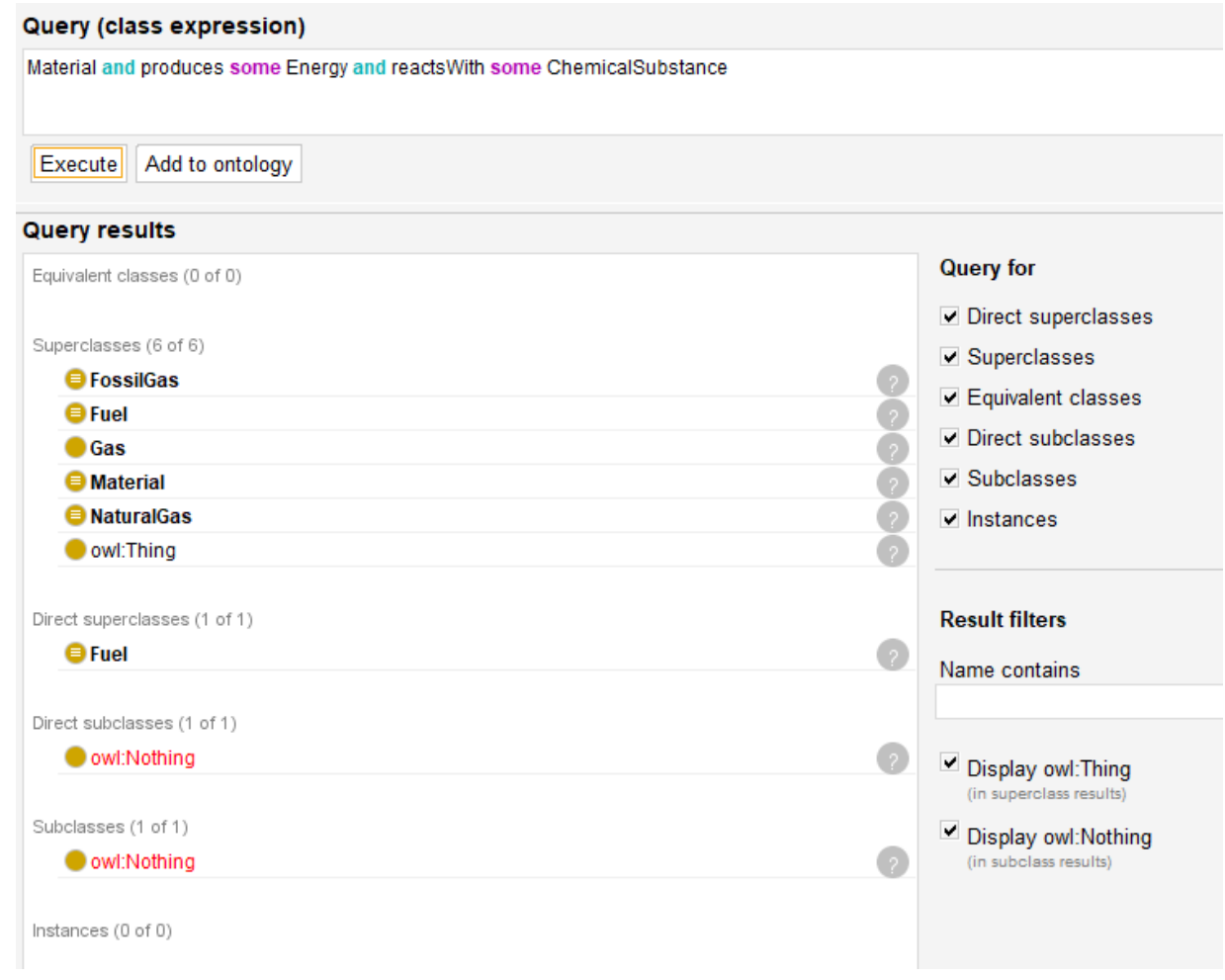

Figure 9. Second query output.

The purpose of this query is to find all types of Material that fulfilled the feature of producing energy and reacting with chemical substances. The query output is compliant with the expected result: the listed materials were analysed by domain experts to verify the correctness of the output.

In Figure 10 the following query has been executed: EconomicSystem and supports some Sustainability

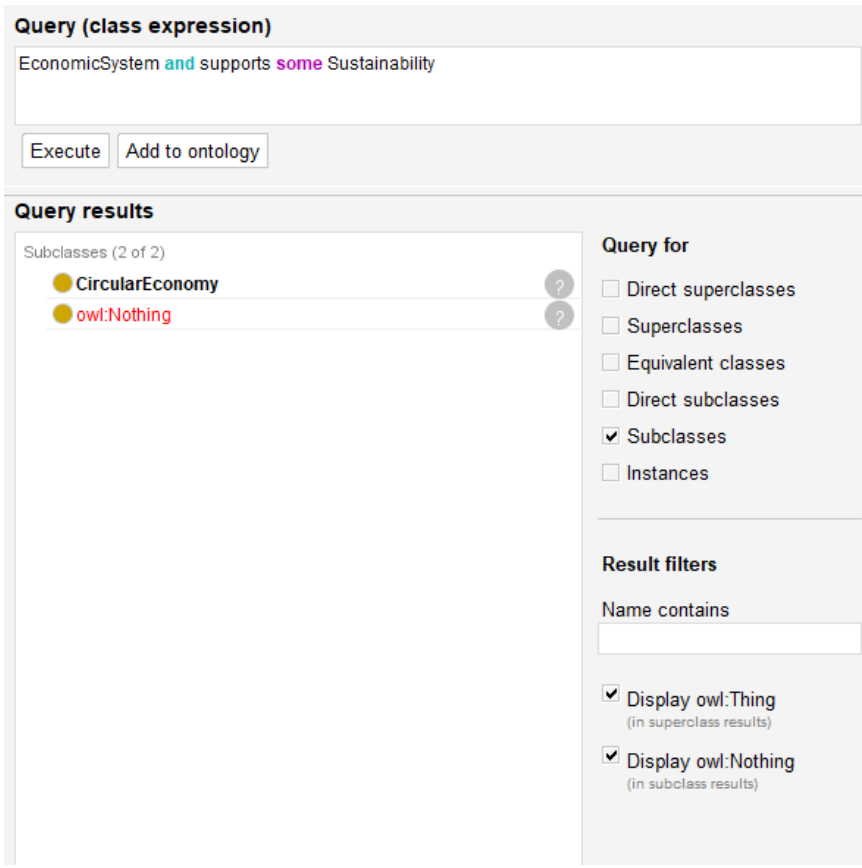

Figure 10. Third query output. 
The purpose of this query is to find which economic system supports sustainability. The query output is compliant with the expected result: as previously mentioned, the circular economy is an economic system and is the means to support sustainability.

\section{Reasoning Process and Design System Search by Ontology (Java Module)}

After the validation process, the BIOVOICES ontology enables the semantic search within the BIOVOICES social platform contents. This module enriches the user's searches via an in-depth analysis of the platform's content: the input keyword is not used to perform a literal match on the content. Still, it improves search accuracy by understanding the searcher's intents and the contextual meaning of the term itself. Therefore, the designed ontology is used to classify and return information facilitating the community members in the search and extraction of information of interest.

As the users of the BIOVOICES social platform can act both as knowledge producers and consumers we designed the semantic module structured in two parts: (i) a content analyser, used when a user produces knowledge and (ii) a semantic searcher used when a user access and consumes knowledge.

The analyser performs the following steps for providing the content analysis:

- Document or event content transformation via Aspose API,

- Concept extraction via TextRazor API,

- Content classification through a comparison with the ontology,

- Final content tagging and backed with a relational database.

This process enables classifying any new content in a semantic way. This classification maps the content in a network of concepts that are periodically updated, enriching the knowledge base of the platform. Every user can upload any content of his/her interest: the platform analyses and tags this content with an automatic approach. The obtained output is a set of tags representing the list of relevant concepts extracted from the new content. Regularly, a domain expert analyses the set of new tags and verifies if, those previously not present in the ontology are significant for the domain of interest. In such a case, the ontology will be updated through the addition of new concepts and relations.

Aiming to support a semantic search, the module performs these steps:

- Keyword search through the ontology concepts' analysis;

- Semantic analysis to extract similar concepts like superclasses, subclasses and equivalent classes;

- Database queries for content matching between tags and extracted concepts;

- Visualisation of the queries results ordered by similarity with the keyword.

The semantic search provides a contextual output: it produces meaningful outcomes even if the query keyword is not present in the retrieved content (see Figure 11).
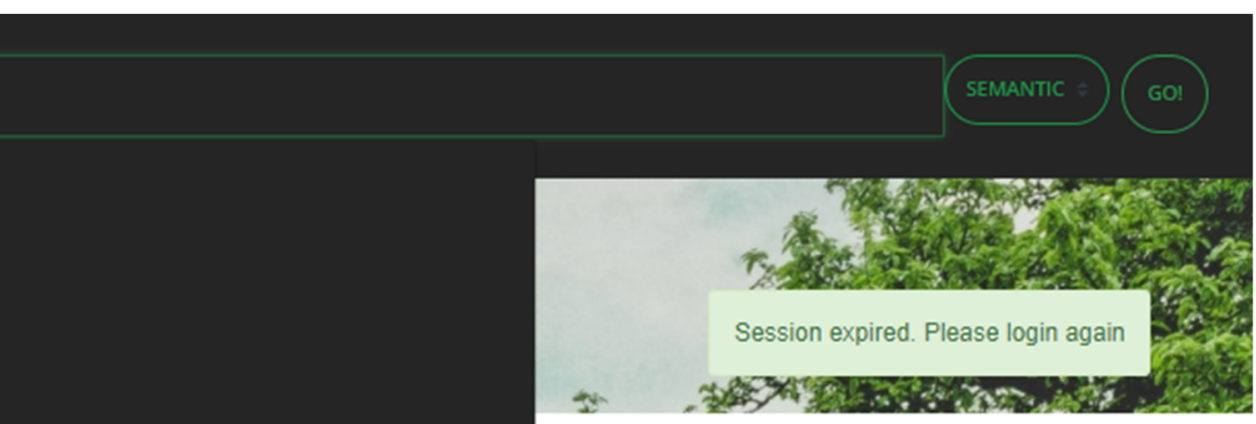

FILTER BIOVOICES CONTENTS!

Figure 11. BIOVOICES platform search interface. 
The module has been developed with Java 8, through the IntelliJ Idea IDE: the communication with the BIOVOICES platform takes place via Rest APIs. This allows reusing the module in different contexts, adapting the configuration to the domain of interest. Moreover, an interface has been developed to use the semantic search module in the BIOVOICES platform.

To make the module usable, it has been developed an auto-fill suggestion functionality which leads users in choosing the right query keyword. We followed the standard guidelines in designing the search interface: it consists of a text box and a button next to it and it is placed on the top of the page, in a navbar, so that it can be reachable from every page of the platform. The output of the search is presented as a list of categorised content (documents, events, mobilisation and mutual learning workshops, etc.) related to the topic of interest. The module, also, shows a list of additional content related to the searched topic, ordered on the basis of its relation.

\section{Conclusions and Future Work}

This article addressed compound problem of strengthen bioeconomy developing a holistic perspective, coherent with the SDGs and actively involving the QH stakeholders.

This requires establishing a common background that can require structuring concepts and relationships connected to the bioeconomy and sustainable bioeconomy. For this reason, we have defined the BiOnto ontology, based on the experience, the information and the services provided in the BIOVOICES multi-stakeholders platform.

The ontology has been built and validated and it facilitates searching and retrieving information shared by the different stakeholders also using semantics.

We have described the steps for defining the taxonomy and then BiOnto ontology and, finally, for validating it. Even if some collections of terms and glossaries are available [11], we did not find structured ontologies in the literature. For this reason, we decided to use a semi-automated approach for defining BiOnto, building a first version that can be extended in future works, including new content that can be shared in a participatory way on the BIOVOICES multi-stakeholder social platform that will be maintained to enable the community to have a common virtual space. Indeed, as future work it is planned to integrate a learning algorithm that will be useful to automatically extend the ontology domain using an agile approach. The use of an agile approach will be adopted to improve the ontology usability considering the different perspectives of the $\mathrm{QH}$ stakeholders.

Author Contributions: Conceptualization, C.B., N.B., F.F., P.G.; methodology, C.B., N.B., F.F., P.G.; software, C.B., N.B.; validation, C.B., N.B., F.F., P.G.; formal analysis, C.B., N.B.; investigation, C.B., N.B.; resources, P.G., F.F.; writing-original draft preparation, C.B., N.B., F.F., P.G.; writing-review and editing, N.B.; supervision, C.B.; funding acquisition, P.G., F.F. All authors have read and agreed to the published version of the manuscript.

Funding: This research was funded by the H2020 Framework Programme, grant number 774331.

Institutional Review Board Statement: Not applicable as the study did not involve humans or animals. Informed Consent Statement: Not applicable.

Data Availability Statement: There are no data for supporting the results of this study.

Conflicts of Interest: The authors declare no conflict of interest.

\section{References}

1. Holden, E.; Linnerud, K.; Banister, D. Sustainable Development: Our Common Future Revisited. Glob. Environ. Chang. 2014, 26, 130-139. [CrossRef]

2. The European Bioeconomy Strategy. 2012. Available online: https://ec.europa.eu/research/bioeconomy/pdf/official-strategy_ en.pdf (accessed on 30 March 2021).

3. Global Bioeconomy Summit (2015). Making Bioeconomy Work for Sustainable Development. Berlin: Global Bioeconomy Summit. Available online: http://gbs2015.com/fileadmin/gbs2015/Downloads/Communique_final.pdf (accessed on 30 March 2021). 
4. Havlík, P.; Leclère, D.; Valin, H.; Herrero, M.; Schmid, E.; Soussana, J.F.; Mueller, C.; Obersteiner, M. Global Climate Change, Food Supply and Livestock Production Systems: A Bioeconomic Analysis. In Climate Change and Food Systems: Global Assessments and Implications for Food Security and Trade; Elbehri, A., Ed.; FAO: Rome, Italy, 2015; pp. 178-197.

5. Linser, S.; Lier, M. The Contribution of Sustainable Development Goals and Forest-Related Indicators to National Bioeconomy Progress Monitoring. Sustainability 2020, 12, 2898. [CrossRef]

6. Prananingrum, L.; Aziz, N.; Wulandari, L.; Banowosari, L.Y.; Suhendra, A. Web Based Ontology Implementation for Information Search System. In Proceedings of the 2019 International Seminar on Research of Information Technology and Intelligent Systems (ISRITI), Yogyakarta, Indonesia, 5-6 December 2019; pp. 499-504. [CrossRef]

7. Kumar, N.A.; Ramu, K. Ontology Based Website for Job Posting and Searching. Conf. Ser. Mater. Sci. Eng. (IOP) 2021, $1042,012006$. [CrossRef]

8. Berners-Lee, T.; Hendler, J.; Lassila, O. The semantic web. Sci. Am. 2001, 284, 34-43. [CrossRef]

9. Pileggi, S.F.; Indorf, M.; Nagi, A.; Kersten, W. CoRiMaS-An Ontological Approach to Cooperative Risk Management in Seaports. Sustainability 2020, 12, 4767. [CrossRef]

10. Sustainable and Circular Bioeconomy for Food Systems Transformation. Available online: http://www.fao.org/in-action/ sustainable-and-circular-bioeconomy/glossary/en/ (accessed on 8 March 2021).

11. Bioeconomy Glossary. Available online: https://knowledge4policy.ec.europa.eu/bioeconomy/glossary_en (accessed on 22 March 2021).

12. Bioeconomy Definition. Available online: https://ec.europa.eu/programmes/horizon2020/en/h2020-section/bioeconomy (accessed on 1 August 2020).

13. Hammack, J.; Brown, G.M., Jr. Waterfowl and Wetlands: Toward Bioeconomic Analysis; Routledge: London, UK, 2016.

14. Núñez-Cacho, P.; Molina-Moreno, V.; Corpas-Iglesias, F.A.; Cortés-García, F.J. Family Businesses Transitioning to a Circular Economy Model: The Case of "Mercadona". Sustainability 2018, 10, 538. [CrossRef]

15. Górecki, J.; Nunez-Cacho, P.; Corpas-Iglesias, F.A.; Molina, V. How to convince players in construction market? Strategies for effective implementation of circular economy in construction sector. Cogent Eng. 2019, 6, 1690760. [CrossRef]

16. Albertini, S.A.; Overbeek, G.; Hoes, A.C. D3.3 Mapping Bio-Based Products (Applications) Based on Stakeholders' Interests. BIOVOICES Project. 2018. Available online: https: / / www.biovoices.eu/download.php?f=35\&l=en\&key=3693dd48b0a5d4d038 ae13697f153d8f (accessed on 30 March 2021).

17. Noy, N.; McGuiness, D. Ontology Development 101: A Guide to Creating Your First Ontology; Technical Report KSL-01-05; Stanford Medical Informatics Technical Report SMI-2001-0880; Stanford Knowledge Systems Laboratory: Stanford, CA, USA, 2001.

18. European Commission Bioeconomy Strategy. A Sustainable Bioeconomy for Europe: Strengthening the Connection between Economy, Society and the Environment; European Commission: Brussels, Belgium, 2018. Available online: https://knowledge4policy.ec. europa.eu/publication/sustainable-bioeconomy-europe-strengthening-connection-between-economy-society_en (accessed on 30 March 2020).

19. TextRazor. Available online: https://www.textrazor.com/docs/java (accessed on 8 March 2021).

20. Jander, W.; Wydra, S.; Wackerbauer, J.; Grundmann, P.; Piotrowski, S. Monitoring Bioeconomy Transitions with EconomicEnvironmental and Innovation Indicators: Addressing Data Gaps in the Short Term. Sustainability 2020, 12, 4683. [CrossRef]

21. Protégé. Available online: https:/ / protege.stanford.edu/ (accessed on 8 March 2021).

22. OWL-Semantic Web Standards. Available online: https://www.w3.org/OWL/ (accessed on 8 March 2021).

23. FluentEditor. Available online: https:/ / www.cognitum.eu/semantics/FluentEditor/ (accessed on 8 March 2021).

24. Musen, M.A. The Protégé project: A look back and a look forward. AI Matters 2015, 1, 4-12. [CrossRef] [PubMed]

25. Samir, T.; Ismailcem, A.; Sheth, A. Ontological Evaluation and Validation. In Theory and Applications of Ontology: Computer Applications; Springer: Berlin/Heidelberg, Germany, 2010. [CrossRef]

26. First Generation Biofuel Definition. Available online: http:/ / biofuel.org.uk/ first-generation-biofuels.html (accessed on 8 March 2021). 